Mini-review

\title{
Network design meets in silico evolutionary biology ${ }^{\star}$
}

Guillermo Rodrigo $^{\mathrm{a},{ }^{*}}$, Javier Carrera ${ }^{\mathrm{a}, \mathrm{b}}$ and Santiago F. Elena ${ }^{\mathrm{a}, \mathrm{c}}$

${ }^{a}$ Instituto de Biología Molecular y Celular de Plantas, Consejo Superior de Investigaciones Científicas-Universidad Politécnica de Valencia, 46022 València, Spain.

${ }^{b}$ Instituto ITACA, Universidad Politécnica de Valencia, 46022 València, Spain.

${ }^{c}$ The Santa Fe Institute, Santa Fe, New Mexico 87501, USA.

Grant support: Supported by fellowships from Generalitat Valenciana (BFPI-2007-160) and the

European Molecular Biology Organization (ASTF-343-2007) to G.R.; and by grants from the Spanish Ministerio de Ciencia e Innovación TIN-2006-12860 to J.C. and BFU2009-06993 to S.F.E.

${ }^{*}$ Corresponding author: Instituto de Biología Molecular y Celular de Plantas, CSIC-UPV, Campus UPV CPI 8E, Ingeniero Fausto Elio s/n, 46022 València, Spain.

E-mail: guirodta@ibmcp.upv.es (G. Rodrigo). 


\begin{abstract}
Cell fate is programmed through gene regulatory networks that perform several calculations to take the appropriate decision. In silico evolutionary optimization mimics the way Nature has designed such gene regulatory networks. In this review we discuss the basic principles of these evolutionary approaches and how they can be applied to engineer synthetic networks. We summarize the basic guidelines to implement an in silico evolutionary design method, the operators for mutation and selection that iteratively drive the network architecture towards a specified dynamical behavior. Interestingly, as it happens in natural evolution, we show the existence of patterns of punctuated evolution. In addition, we highlight several examples of models that have been designed using automated procedures, together with different objective functions to select for the proper behavior. Finally, we briefly discuss the modular designability of gene regulatory networks and its potential application in biotechnology.
\end{abstract}

Keywords: evolutionary optimization, regulatory network, systems biology, synthetic biology.

\title{
1. Introduction
}

Over the last two decades, mathematical and computational techniques have been widely applied in biology aiming to understand how cells behave and differentiate as the result of tens of thousands of molecular interactions [1]. The challenge of Systems Biology is to describe these interactions in precise quantitative mathematical terms that allow making new predictions about how cells may respond to external and internal perturbations [2]. These models are thus based on first-principles and experimental observations. Furthermore, this detailed knowledge should be rapidly available to be used in the design of new genetic systems and reengineered cells by combining different of such model pieces (Table 1). This attempt for modular reprogramming cell functions takes advantage of improved recombinant DNA techniques and model-based approaches, and such an approach constitutes the emerging field of Synthetic Biology [3]. But, how can we design new circuits? 
Rational model-guided approaches have turned out to be very fruitful for engineering standardized genetic modules for biotechnological applications from a library of characterized promoters [4]. However, such engineering will require the use of optimization techniques aiming to explore the endless combinatorial space represented by all possible solutions. What would be the most efficient approach for such tasks? Could we use automated methods to design biological circuits?

Natural genetic circuits have evolved and have been optimized for their function, while being robust to perturbations (e.g., environmental changes or genetic mutations), by means of natural selection. Can we use the same principle to create de novo genetic circuits? Indeed, evolution can be seen as an adaptive walk in a genotypic landscape. Errors during the replication of genomes (all sort of mutations, including recombination) allow populations to reach distant areas of the landscape, and those individuals in the population having the best genomes will contribute with more descendants to the next generation than those which are less fit. Such a difference in reproductive success is the ground for natural selection to operate $[5,6]$. This is a recurrent trialand-error process where random mutations that occur in the genome may change the cellular program. Then, according to a given fitness function (e.g., the simplest one being the number of descendants produced by a genotype relative to the average number of descendants of the population) the fitter organisms increase their frequency in the population and, thus, the population moves towards local optima in the fitness landscape acting as attractors [6].

In silico evolution mimics the process described above throughout a heuristic search in which a system is iteratively modified (mutation process) and evaluated for its performance towards a predefined target (fitness computation). Depending on the size and complexity of the network to be evolved, large-scale and high performance computational facilities would be required to allow for the computation of many generations. In this review we focus on the use of evolutionary optimization algorithms as a design tool to engineer synthetic regulatory circuits with specified functionalities [7], although such an approach may also offer the possibility to evolutionary biologists of analyzing the validity of their models of genomic evolution and learning about the 
design principles for intracellular organization [8]. We highlight different key aspects for the computational implementation of evolutionary techniques and present illustrative examples. Although evolutionary techniques have been widely applied for parameter optimization in other research areas, little has been done for the de novo design of biological circuits accounting for dynamical features.

\section{Optimization schemes that mimic natural evolution}

Over the last decade, the algorithms based on probabilistic schemes that mimic natural evolution have been used to address optimization problems (Fig. 1). Depending on the implementation of the iterative process of "mutation-then-selection", we can describe a wide range of Evolutionary Algorithms (EAs) [9,10]. EAs are a popular family of optimization methods inspired in the biological principles that govern the evolution of a finite population through certain selective pressure (e.g., the ability to perform a given task). Random mutations occur in the descendants (in this case in the form of alterations in the network) and only those exhibiting the largest efficiency in performing the target task are selected for the next generation. In the next generation, these individuals reproduce proportionally to their fitness; with new mutations happening during the application of the reproduction operators at a certain rate, thus repeating the process.

The difference between several EAs is more a question of implementation. The reproduction operators and the selection procedure can be parameterized obtaining a wide range of algorithms depending on the values of a set of control parameters. In network design, instead of evolving a sequence of bits, the way of generating offspring consists in modifying the nodes (genes) and edges (regulations) of the parent network by mutation and/or crossover. Although the EA essentially becomes hill-climbing, we have also to notice that the common use of the reproductive operators as a generator of diversity around one generation allows the populations to perform sporadic random jumps over the fitness surface towards nearby peaks (Fig. 1a). Certainly, crossover allows for rapidly traversing large valleys by combining beneficial information from the parents $[11,12]$. 
However, when crossover does not confer a selective advantage or its occurrence is not significant relative to the occurrence of single point mutations, the evolution of the population can be viewed as multiple individuals evolving in parallel.

The selection method constitutes the major element in an EA, as the designed network will be the result for what we have selected. In principle, the best performing individuals are selected at each step (e.g., $10 \%$ of the population). For example, the Elite Selection strategy consists in retaining the best individuals thwarting their replication without further mutation nor recombination, ensuring that the offspring is at least as good as their parents $[9,10]$. A more complex approach is represented by the Wright-Fisher (synchronous reproduction) or the Moran (asynchronous reproduction) processes [13], for which competition among individuals occurs in a spatial context. However, we have to notice that a strong pressure reduces the diversity required for a quick adaptation to other environments, whereas a weak pressure results in a too slow optimization process. Another inconvenient of EAs is a premature convergence into local optima after only very few generations. In the Boltzmann criterion, the initial evolutionary dynamics closely resembles a random walk (and hence a more efficient exploration of the fitness surface), whereas as time goes on the dynamics resembles an adaptive walk. Herein, the logarithm of the probability of accepting an individual for reproduction is proportional to its fitness and inversely proportional to the "temperature" of the system. The parameter temperature controls the size of the evolutionary steps, from a perfect random walk (at very high temperatures) to a pure adaptive walk (at very low temperatures). Typically, temperature follows a user-defined cooling function during the evolution (e.g., an exponential decaying function with a very high starting temperature). Thereby, Monte Carlo Simulated Annealing [14], an algorithm mostly used for optimization in engineering, can be viewed as another EA to evolve one individual or even a population [15].

\section{Fitness landscape in network design}

The characteristics of the landscape strongly condition the performance of the EA and, thus, the 
choice of the parameters that characterize a given evolutionary optimization technique [11]. One important property is the ruggedness [16], that is, the magnitude of the change in fitness for a small movement in the landscape. To illustrate this property in gene network design, we have performed a random sampling for a test problem. We applied our algorithm [17] to optimize a transcription circuit behaving as an AND logic gate with two repressors as inputs on different promoters. Herein, we considered two mutation operators: one to evolve the circuit in the parameter space and another to evolve the topology. A parameter change was 9-fold more likely than a change in topology (i.e., the $90 \%$ of the moves were in the parameter space for a fixed topology). We observed that the fitness distribution is highly skewed (Fig. 2a), giving an intuitive idea of the distribution of local optima in the landscape: very few local optima exist embedded in a large region of inefficient circuits (isolated peaks) [11]. To have a quantitative measure of the ruggedness, we have calculated the fitness autocorrelation function (Fig. 2b). As expected for rugged landscapes, in average the similarity in fitness between two points from a random walk decreases with the number of steps necessary to go from one point to another. We can appreciate that this decrease is, in average, uniform with the number of steps, although some particular runs showed a punctuated pattern in which stasis periods were interrupted by short periods of large decreases (data not shown). Then, according to the principle of strong causality (where small changes in the network parameters produce, in average, small changes in its dynamics), it will be easy to construct an evolutionary path towards a good solution ensuring the convergence of the algorithm [18]. Fig. 2c shows the fitness evolution for a random walk versus an adaptive walk using a Boltzmann criterion. The Figure illustrates a general property of adaptive systems, namely the existence of patterns of punctuated evolution in which the populations spend most of their time drifting on neutral areas of the fitness landscape and important evolutionary events occur very fast on time [19]. This irregular shape of the landscape poses in many cases several difficulties for heuristic algorithms and a good landscape should avoid this by rewarding intermediate regions in fitness. 
The mathematical model and the fitness function have to be constructed in such a way that organisms close to the target objective, for instance a specific dynamic behavior, gain an advantage. Hence, the landscape will be less rugged facilitating the search of the EA. When doing so, one usually specifies the dynamics for the inputs and the desired behavior for the outputs. However, selective pressures over genes that are not directly involved in the fitness function (auxiliary genes) could be minimal or even inexistent, that is, they behave as neutral loci. However, due to an inappropriate election of the fitness function, these auxiliary genes may exert some unexpected pleiotropic effect over other genes in the cellular background and thus impact the dynamics of the output genes in undesired and unpredictable ways. In addition, the initial condition is also pivotal for optimal design, since it conditions the network dynamics. For instance, in case of aiming the design of a multistable circuit that can be switched by external stimuli, the designer has to incorporate all different dynamic transitions from all possible initial states. Otherwise, the system will not develop the hysteresis mechanism necessary for a memory-like circuit [20].

\section{Guidelines to construct the evolutionary optimization}

The first step to be met in such an evolutionary procedure consists in defining the different types of interactions, such as transcriptional, post-translational or metabolic, their relationships and their mathematical representation. The model can be deterministic or stochastic. The second step is to define the rules for addition, deletion and replacement of elements in the network, that is, the possible mutations. In network design, the type of mutation (a change in the value of a parameter or an alteration in the topology) determinates the step size in the fitness landscape. A perturbation in one kinetic parameter of the network generally is less dramatic than a change in the topology. Thereby, one elementary optimization strategy consists in a local exploration of the parameter space for a given topology. To account for this difference, the probability for mutating a parameter is set much higher than that for the topology. Moreover, in network design the solution space can be constrained by imposing limited resources (e.g., specifying the maximum number of genes in the 
network). The third point is to define the starting network, which may or may not be random. If no a priori knowledge exists, then a random starting network should be assumed. However, if the goal of the process is to optimize an already existing circuit (or part of it) the initial network must be defined accordingly. Depending on the flexibility of movements in the landscape, the resulting network may or may not resemble the starting one. The final element to be defined is the fitness function to be used for selecting the best network at each step in the optimization process. Different approaches exist to evaluate the relative fitness of an evolving network. Generally, one is interested in achieving certain dynamic gene expression profile by a network that will be engineered to reprogram a given cellular function [3]. In that way, a mathematical description (deterministic or stochastic) allows monitoring at each time point during the optimization process the dynamical response of the evolving network relative to the desired target. Usually, the magnitude used to compare dynamical responses consists in a weighted Euclidean distance that gives a measure of the closeness between two dynamics [17]. The weighting factor dictates the time region for the fitness computation, for instance, to avoid transients. More flexible approaches are functions based on temporal logics, where the qualitative traits (e.g., up to a threshold) are the relevant features contributing to fitness [21]. Statistical regression functions can also be used to correlate two dynamics [22]. As an alternative to dynamics-based functions, the spectral analysis provides new insights for approaching fitness functions. Indeed, by exploiting the spectrum obtained from the mathematical model (e.g., an oscillatory system has complex eigenvalues), the fitness function can account for qualitative dynamical behaviors [23]. Finally, in case one may decide to neglect the dynamical features of a network and just focus on its topology, Hamming-like distances are useful tools to compare between pairs of topologies [24]. In Table 2 we present different mathematical expressions for fitness computation based on the temporal protein expression profiles. After all elements have been defined, the evolutionary algorithm can be applied for a defined number of generations or until the target is reached. 


\section{Illustrative examples: de novo design of networks}

One of the most successful applications of rational design techniques [25] was the synthetic oscillatory circuit named "repressilator" engineered by Elowitz and Leibler in Escherichia coli [26]. This synthetic regulatory network consisted of three transcriptional repressors (lacI, tetR and $\lambda c I$ ) disposed in a ring and coupled to the GFP reporter. The resulting circuit had a predictable behavior and the mathematical model allowed identifying the parameter space favoring oscillations.

Being that, could model-guided automated methods look for designs like these? On the one hand, François and Hakim [7] designed different biological clocks relying on transcription and posttranslation regulations. They used a genetic algorithm with $\sim 100$ cells together with a scoring function based on the signal period between two arbitrary levels (on/off). The network dynamics was only evaluated in a neighborhood of maxima and minima over 10 periods, and the solution was found after $\sim 300$ generations. Such an approach was found to be more efficient than using sophisticated methods based on Fourier analysis. In comparison to naturally occurring circuits, they found that a negative feedback loop was primary responsible of the oscillatory behavior. In addition, this evolutionary strategy allowed the suggestion of different working principles beyond what was already known for circadian clocks depicted by high-nonlinear and/or delay-based models. On the other hand, we used an EA with a Boltzmann criterion, following a similar scoring procedure as described above, to design oscillatory circuits that responded to different forcing cyclic stimuli [27]. The clocks we evolved were autonomous (i.e., oscillations without an external signal) or phase-locked to the cycle period. Roughly, we found circuits able to adapt their rhythms according to the forcing stimulus, whereas other circuit dynamics were not altered by the external influence maintaining their free-running periods.

Further work on de novo design based on evolutionary principles has resulted in a very useful, wide palette of functional regulatory circuits [20,22,23,28] and even genome-scale metabolic networks [24] implementing a targeted behavior. In particular, Sauro's group developed an EA to design functional networks (metabolic and genetic) able to implement mathematical calculations, 
oscillators, memories, and frequency filters [23]. Strikingly, Tavazoie and colleagues suggested a given ability by microorganisms to anticipate changes in their environment that allowed them to adjust their physiological state prior to experiencing the environmental challenge, ensuring a more efficient and rapid consumption of newly available resources [22]. To confirm in silico this observation, they used an EA, with stochastic events, representing a population of cells subjected to fluctuating environments with the corresponding resources. They found that such a predictive behavior is implemented by means of redundant gene networks (i.e., highly wired networks). One more example. In silico evolution procedures can approach the study of embryonic morphogenesis by engineering pattern formation. In this case a set of independent cells located in different spatial points with the same gene network sense a gradient that triggers a pattern. Herein, the fitness function favors, for instance, the number of segment boundaries [29]. Such an approach can be applied to design user-specified biological patterns, and the features of the resulting networks may be extrapolated to understand the natural organization of living systems.

\section{Trade-offs in network design}

The question of how good is our design leads the à la carte design of genetic and metabolic circuits, that is, with multiple specifications. As in other engineering fields, the systems to be designed are subjected to specifications that usually present trade-offs. For instance, one may be interested not only in improving the overall metabolic flux but also making the process more robust against external perturbations or with modularity properties to facilitate its use as a standard for future projects. The design of robust networks is certainly a challenge in Synthetic Biology and it would constitute by itself the matter of a prospective study. As it has been previously reported [30], robustness is a ubiquitous property of biological systems that arise from the precise network structure, where the steady state of a system's variable (a concentration or a rate) is invariant under perturbations. Robustness is generally related to critical properties for the appropriate functioning mode and, for each system, can be associated to design principles of biological networks [2]. 
Although an exhaustive description of methods to design robust networks goes beyond the scope of this Minreview, herein we provide some insights on how to approach the design of networks by optimization that can be insensitive to variations in the kinetic parameters. Moreover, these could straightforwardly extended to account for variations in the structural properties of the network (i.e., topological changes), in which networks will be a priori redundant to sustain the elimination of a node or edge. We therefore succinctly point up the exploitation of EAs to generate multi-functional gene regulatory networks.

The additional constraints in the design may be imposed into the fitness function. For example, the definition of different evolutionary targets combinatorially constructed from a set of subtargets spontaneously boosts a modular organization that allows for rapidly adopting the appropriate operative mode [31]. Nonetheless, multi-objective optimization is a hard task; especially if trade-offs among different characters exist. For illustrative purposes, let us consider the case of designing a functional and robust gene network. Certainly, for limited resources, an increase in robustness provokes a reduction in performance [30]. Then, the solution may be not unique and it will depend on the design specifications (e.g., how much robust must be the design). One strategy to address this optimization constrain is by constructing a weighted convex sum of objectives (Pareto-optimal front). According to different values of these weighting factors (i.e., giving more importance to one objective than to another), we recover the specific virtual landscape for the problem. The incorporation in the fitness function of a weighted term that accounts for the change in the system behavior after losing one element serves to evolve regulatory networks displaying certain degree of robustness [32]. In addition, the use of dynamic weights (e.g., periodic functions) allows improving, especially in high-dimension systems, the efficiency of the EA to reach optimal solutions satisfying all objectives [33].

An alternative to multi-objective optimization consists in designing a survival function that rewards networks satisfying all objectives. In case of robust design, the fitness function, which only accounts for performance, is evaluated at each step during the evolution process in a nearby 
point in the landscape. A single dynamic perturbation is often more efficient than averaging several of the neighbors [32]. Thereby, robust networks will be naturally selected without a pressure for that, contrarily as it occurs in multi-objective optimization. Moreover, the multiple design specifications can be approached in terms of independent factors that act in a successive manner. In that way, regulatory circuits are designed to adapt the cell behavior to a changing environment with a dynamic fitness function. Kashtan et al. [33] serves as a good illustrative example. These authors used a digital representation for gene interactions together with a Boolean fitness calculation, where the objective function changed after a specified number of generations, to design circuits with the capacity of adaptation to different environments (Fig. 1b). In addition, they found that, under certain circumstances, the use of dynamic fitness landscapes could speed up the evolutionary design.

\section{Redesign of preexisting cellular networks}

The construction of accurate predictive models not only allows us to monitor the dynamic features of biological networks, but also to study the effect of different perturbations on the behavior of the system. In addition, with the advent of high-throughput "omic" technologies, it becomes possible for the first time since the origins of molecular biology the construction of genome-scale models. Although we are still far from the complete description of the intracellular organization in mathematical terms, reverse-engineering methods have been successfully applied to these massive data for reconstructing models of the networks of genetic interactions that have allowed predicting cellular responses to external perturbations [36] as well as accounting for mutations that alter the network structure [37,38]. However, it is difficult to determine whether these models, global or not, reflect the reality or they are just artificial constructions that allow us to study the dynamical properties of biological networks. Being the second case, the starting networks would not offer the possibility of extrapolating any insight obtained by manipulating the model.

In case of having a reliable network model, the next logical development will be to use these 
predictive models as substrates for reengineering networks in such a way that the resulting cell would show a new or improved phenotype of biotechnological interest. Recently, Isalan and coworkers [39] experimentally rewired bacteria by up-regulating wild-type transcription factors using natural promoters, finding that some of the new regulations were maintained by the cell under different selective pressures. What are the advantages that offer new regulations? Using modelbased approaches we can address this question for a few specific interactions [38], but EAs can explore the vast combinatorial space and propose optimal rewiring of natural systems. In this sense, evolutionary methods can be used to redesign the natural cell organization in such a way that certain regulatory networks or metabolic pathways (or why not, the entire cell interactome) would be optimized for a target phenotype (Fig. 1c).

\section{Concluding remarks}

Using the designing framework here presented, we have illustrated how to evolve in silico regulatory circuits from individual genes or even the whole genome organization. The evolutionary optimization mimics the way Nature has designed genetic networks. To implement this process, we use stochastic search algorithms for efficiently exploring the fitness landscape defined by all possible network designs. The shape of the landscape is given by the objective function used, which leads the optimization (selection and convergence) of functional regulatory networks. From a practical perspective, these newly designed functional modules can be obtained from already existing libraries of standard interchangeable parts [40] and encoded in synthetic DNA cassettes and carried in any sort of delivery vector for further genomic assembly. Certainly, evolutionary optimization explores a large amount of combinations of such counterparts under a user-specified objective function. Moreover, the ability of fully synthesizing/assembling those cassettes and the corresponding implementation into a cellular chassis opens the door to new horizons for biotechnology [41]. For example, of particular interest are reengineered bacterial strains expressing heterologous enzymes for biofuels or pharmaceuticals production [42]. Remarkably, the engineered 
regulations that may provide an additional advantage to the cell will therefore have increased chances of being maintained for the next generations.

The cellular background of operation limits the elements and mechanisms by which constructing a synthetic network with a dynamical function. It is almost clear that in higher organisms the degree of sophistication and fine-tuning of the systems poses particular challenges for prediction and for a proper function because the potential interactions with the large amount of embedded molecules. In that way, bacteria, and even yeast, are well-characterized organisms to accommodate the designed networks. In prokaryotes, it is generally undertaken a decoupling between the promoter and coding regions; thus, despite certain exceptions, allowing a modular composition between regulatory and regulated genes. In addition, the organization of genes into operons let us assume same transcriptomic level for all the genes belonging to a given operon. However, in eukaryotes transcription involves many macromolecules and the elongation process is much complex because splicing (e.g., a single gene can give different proteins depending on the introns removed) [43]. Therefore, orthogonal systems may represent an optimal solution since they allow us to overcome the distinctive machinery of each organism [44], such as transcription factors, RNA-polymerases or ribosomes. These systems that work in parallel to the normal cellular machinery may produce a lower cost to the cell because the resources can be dedicated for the rest of its functions. All in all, the engineered cells with synthetic circuitries will result in micromachines working, for instance, as photosystems, drug deliverers, material weavers, or biofactories.

\section{References}

1. H. Kitano, Computational systems biology, Nature 420 (2002) 206-210.

2. U. Alon, An Introduction to Systems Biology: Design Principles of Biological Circuits, Chapman \& Hall/CRC, Boca Raton FL, 2006. 
3. D. Sprinzak, M.B. Elowitz, Reconstruction of genetic circuits, Nature 438 (2005) 443-448.

4. T. Ellis, X. Wang, J.J. Collins, Diversity-based, model-guided construction of synthetic gene networks with predicted functions, Nat Biotechnol 27 (2009) 465-471.

5. G.C. Williams, Adaptation and Natural Selection, Princeton University Press, New Jersey, 1996.

6. S.F. Elena, R.E. Lenski, Evolution experiments with microorganisms: the dynamics and genetic bases of adaptation, Nat Rev Genet 4 (2003) 457-469.

7. P. François, V. Hakim, Design of genetic networks with specified functions by evolution in silico, Proc Natl Acad Sci USA 101 (2004) 580-585.

8. A.P. Quayle, S. Bullock, Modelling the evolution of genetic regulatory networks, J Theor Biol 238 (2006) 737-753.

9. M. Mitchell, An Introduction to Genetic Algorithms, MIT Press, Cambridge MA, 1996.

10. J.H. Holland, Adaptation in Natural and Artificial Systems, MIT Press, Cambridge MA, 1992.

11. J.H. Holland, Using classifier systems to study adaptive nonlinear networks, In D.L. Stein (ed.) Lect Sci Complex, Addison-Wesley, Reading MA, 1989.

12. M. Mitchell, S. Forrest, J.H. Holland, The royal road for genetic algorithms: fitness landscapes and GA performance. In Toward a Practice of Autonomous Systems: Proc I Eur Conf Artificial Life, MIT Press, Cambridge MA, 1992.

13. W.J. Ewens, Mathematical Population Genetics I. Theoretical Introduction, Springer Verlag, NewYork, 2004.

14. S. Kirkpatrick, C.D. Gelatt, M.P. Vecchi, Optimization by Simulated Annealing, Science 220 (1983) 671-680.

15. K.P. Wong, Y.W. Wong, Genetic and genetic/simulated-annealing approaches to economic dispatch, IEE Proc Gener Transm Distrib 141 (1994) 507-513.

16. E.D. Weinberger, Correlated and uncorrelated fitness landscapes and how to tell the difference, Biol Cybern 63 (1990) 325-336. 
17. G. Rodrigo, J. Carrera, A. Jaramillo, Genetdes: automatic design of transcriptional networks, Bioinformatics 23 (2007) 1857-1858.

18. I. Rechenberg, Evolutionsstrategie, Frommann-Holzboog Verlag, Stuttgart, 1994.

19. S.F. Elena, V.S. Cooper, R.E. Lenski, Punctuated evolution caused by selection of rare beneficial mutations, Science 272 (1996) 1802-1804.

20. G. Rodrigo, A. Jaramillo, Computational design of digital and memory biological devices, Syst Synth Biol 1 (2007) 183-195.

21. G. Batt, D. Ropers, H. de Jong H, et al., Validation of qualitative models of genetic regulatory networks by model checking: analysis of the nutritional stress response in Escherichia coli, Bioinformatics 21 (2005) i19-i28.

22. I. Tagkopoulos, Y. Liu, S. Tavazoie, Predictive behavior within microbial genetic networks, Science 320 (2008) 1313-1317.

23. S.R. Paladugu, V. Chickarmane, A. Deckard, et al., In silico evolution of functional modules in biochemical networks, IEE Proc Syst Biol 153 (2006) 223-235.

24. A. Hintze, C. Adami, Evolution of complex modular biological networks, PLoS Comput Biol 4 (2008) e23.

25. M.E. Wall, W.S. Hlavacek, M.A. Savageau, Design of gene circuits: lessons from bacteria, Nat Rev Genet 5 (2004) 34-42.

26. M.B. Elowitz, S. Leibler, A synthetic oscillatory network of transcriptional regulators, Nature 403 (2000) 335-338.

27. G. Rodrigo, J. Carrera, A. Jaramillo, In silico evolution of the oscillatory response under lightdark cycles, Biochimie 90 (2008) 888-897.

28. M.S. Dasika, C.D. Maranas, OptCircuit: an optimization based method for computational design of genetic circuits, BMC Syst Biol 2 (2008) 24.

29. P. François, V. Hakim, E.D. Siggia, Deriving structure from evolution: metazoan segmentation, Mol Syst Biol 3 (2007) 154. 
30. H. Kitano, Biological robustness, Nat Rev Genet 5 (2004) 826-837.

31. N. Kashtan, U. Alon, Spontaneous evolution of modularity and network motifs, Proc Natl Acad Sci USA 102 (2005) 13773-13778.

32. J. Maciá, R.V. Solé, Distributed robustness in cellular networks: insights from synthetic evolved circuits, J R Soc Interface 6 (2009) 393-400.

33. Y. Sin, T. Okabe, B. Sendhorf, Adapting weighted aggregation for multi-objective evolution strategies. Proc I Int Conf Evol Multi-Crit Optim, Lect Not Comp Sci 1993 (2001) 96-110.

34. S. Tsutsui, A. Ghosh, Genetic algorithms with a robust solution searching scheme, IEEE Trans Evol Comput 1 (1997) 201-208.

35. N. Kashtan, E. Noor, U. Alon, Varying environments can speed up evolution, Proc Natl Acad Sci USA 104 (2007) 13711-13716.

36. R. Bonneau, Learning biological networks: from modules to dynamics, Nat Chem Biol 4 (2008) 658-664.

37. G.W. Carter, S. Prinz, C. Neou, et al., Prediction of phenotype and gene expression for combinations of mutations, Mol Syst Biol 3 (2007) 96.

38. J. Carrera, G. Rodrigo, A. Jaramillo, Model-based redesign of global transcription regulation, Nucleic Acid Res 37 (2009) e38.

39. M. Isalan, C. Lemerle, K. Michalodimitrakis, et al., Evolvability and hierarchy in rewired bacterial gene networks, Nature 452 (2008) 840-845.

40. B. Canton, A. Labno, D. Endy, Refinement and standardization of synthetic biological parts and devices, Nat Biotechnol 26 (2008) 787-793.

41. T.K. Lu, A.S. Khalil, J.J. Collins, Next-generation synthetic gene networks, Nat Biotechnol 27 (2009) 1139-1150.

42. C.H. Martin, D.R. Nielsen, K.V. Solomon, et al., Synthetic metabolism: engineering biology at the protein and pathway scales, Chem Biol 16 (2009) 277-286.

43. K. Struhl, Fundamentally different logic of gene regulation in eukaryotes and prokaryotes, Cell 
98 (1999) 1-45.

44. W. An, J.W. Chin, Synthesis of orthogonal transcription-translation networks, Proc Natl Acad Sci USA 106 (2009) 8477-8482. 


\section{Figure Caption and Tables}

Fig. 1: (a) Evolutionary trajectories of networks over the fitness landscape. We plot the evolutionary trajectory of two simulations reaching different network conformations at each step. The difference between these two simulations strives in the fitness function (yellow for designing performing networks, red for performing and robust networks). The initial circuits were randomly chosen in both cases. (b) In silico evolution in changing environments. We show the evolutionary path of a system that is the result of a dynamic fitness function dictated by three different environments. (c) The combination of different quantitative experimental techniques including large "omics" datasets allows developing mathematical models of natural networks, which can be the input of evolutionary methods leading the exploration of several rewirings to redesign the cellular network for exhibiting a new phenotype.

Fig. 2: Fitness landscape sampling using a random walk. (a) Distribution of fitness. Mean, 0.0190 and standard deviation, 0.0156. (b) Fitness autocorrelation. (c) Fitness evolution for a random walk (gray) and for an adaptive walk with a Boltzmann criterion (black). 
Table 1: Scheme of mathematical model construction of natural/synthetic nucleic acids able to interplay between them to yield gene networks. Using experimental data, models are validated or refined for a more accurate prediction. 
Table 2: Possible objective function expressions $(\varphi)$ for minimization to evolve biological networks. To calculate fitness, we can use the transformation $1 /(1+\varphi)$.

Dynamic function ${ }^{\mathrm{a}, \mathrm{b}}$

Temporal logic function ${ }^{\mathrm{a}, \mathrm{c}}$

Spectral function ${ }^{\mathrm{d}}$

Correlation function ${ }^{\mathrm{a}, \mathrm{e}}$

Topological function ${ }^{\mathrm{f}}$
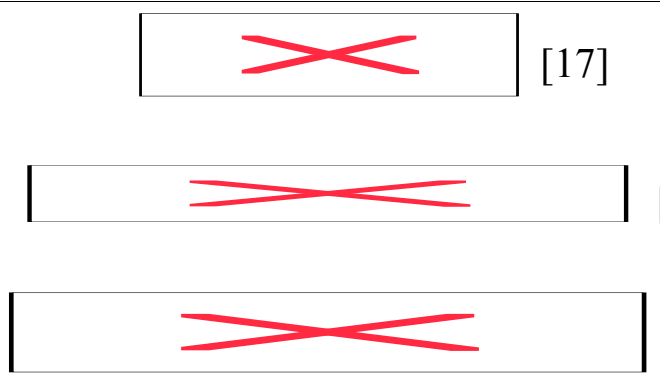

$\varphi=\sigma_{y, z} / \sqrt{\sigma_{y}^{2} \sigma_{z}^{2}}[22]$

${ }^{a} y$ represents the system dynamics and $z$ the specified dynamics.

${ }^{\mathrm{b}} \chi(t)$ is a weighting factor.

${ }^{\mathrm{c}} F(x)$ is a Boolean function.

${ }^{\mathrm{d}}$ Expression to find Hopf bifurcations; $\lambda_{i}$ are the eigenvalues of the system.

${ }^{\mathrm{e}} \sigma$ is the standard deviation. This function is for maximization.

${ }^{\mathrm{f}} d$ is a metric function (e.g., Hamming distance) between two gene patterns or sequences. $S(N)$ is a function of network $N . S_{0}$ is the reference. 